\title{
A Novel Scrambling Digital Image Watermark Algorithm Based on Double Transform Domains
}

\author{
Taiyue Wang ${ }^{1}$ and Hongwei $\mathrm{Li}^{2}$ \\ ${ }^{1}$ School of Mathematics and Physics, Hubei Polytechnic University, Huangshi 435003, China \\ ${ }^{2}$ School of Mathematics and Physics, China University of Geosciences, Wuhan 430074, China \\ Correspondence should be addressed to Taiyue Wang; wangty6895@126.com
}

Received 21 January 2015; Accepted 20 August 2015

Academic Editor: Konstantinos Karamanos

Copyright (C) 2015 T. Wang and H. Li. This is an open access article distributed under the Creative Commons Attribution License, which permits unrestricted use, distribution, and reproduction in any medium, provided the original work is properly cited.

\begin{abstract}
Digital watermark technology is a very good method for protecting copyright. In this paper, in terms of requisition of imperceptibility and robustness of watermarking, the multidirectional, multiscale, and band-pass coefficient features of Curvelet transform are introduced and a novel image watermark scheme based on Curvelet and human visual system is proposed. Digital watermark information is embedded into the first 16 directions with larger energy in the fourth layer. Experimental results indicate that the proposed watermark scheme is feasible and simple. Simultaneously, the embedded watermark images just have tiny difference with the original images and the extracted watermark is accurate. Moreover, it is imperceptible and robust against various methods of signals processing such as cropping, noise adding, and rotating and altering.
\end{abstract}

\section{Introduction}

With the development of multimedia technology and network communications, digital products are increasingly popular. Due to illegal copying and tampering of digital product, traditional encryption technology is not sufficient to protect legitimate rights of digital media copyright. Digital watermarking which is hidden in digital products can play an important role. Recently, the digital watermarking has become one of research hotspots in the field of signal processing and information security. Its key is to embed the unremovable watermarking into the protected original signal without affecting data availability. Simultaneously, the watermarking can be completely and exactly extracted or detected, to satisfy solving the copyright disputes such as piracy tracking, and so forth. Therefore, the embedded digital watermarking must possess the following characteristics $[1$, 2]: (1) imperceptibility: embedded watermarking should not lead to obvious visual difference and hidden information cannot be perceived easily; (2) robustness: the carrier image is attacked or processed; the embedded digital watermark information also can be extracted exactly; (3) maximum of information: the embedded watermark information should be capacity as much as possible in the digital products to achieve the largest hidden information volume of carrier object; (4) determinacy: the copyright of digital watermark information could uniquely determine the owner of the digital products even via certain damage.

According to the difference of embedding watermark technology, the algorithms were divided into watermarking in spatial domain and watermarking in transform domain [3]. For watermarking in spatial domain, the amplitude of sample points of original image was changed directly and its representative algorithm was the Least Significant Bit algorithm [4]. Changing coefficients of carrier image via embedding watermark information was called watermarking in transform domain. In general, the main idea of watermark algorithm in transform domain is changing the image transform domain coefficients to embed watermarking which adopts the spread spectrum communication principle. Digital watermark technology in transform domain has obvious advantages [5, 6]: (1) The watermark information can be distributed into all pixels of spatial domain and ensure the imperceptibility of watermarking. (2) Centralization of energy distribution can embed large quantity of watermark data and guarantee better imperceptibility, 
stronger robustness, and higher security. (3) It is compatible with the existed image compression method and can embed watermarking into the compressed image. (4) It is compatible with human visual system and can hide the watermarking easily. The existed watermark algorithms in transform domain mainly include Discrete Fourier Transform (DFT) [7], Discrete Cosine Transform (DCT) [8], and Discrete Wavelet Transform (DWT) [9]. They can effectively represent point singularity and deficiency for linear or curve singularity of natural images. Recently, with the development of the multiscale geometric transform, the approximation abilities of signal and image are continually enhanced, which is gradually correspondent to the human visual system and has been widely applied in many fields. There are plenty of researches in the field of information security, such as watermark algorithm based on Ridgelet Domain [10] and Curvelet Domain [11]; they can further improve the imperceptibility and robustness of watermark algorithm. According to various transforms and HVS, the capacity of the watermark insertion was introduced in some literatures [12-14].

In this paper, we study an algorithm in transform domain. According to the requirement of digital watermarking robustness and imperceptibility, the multidirectional, multiscale, and band-pass coefficient features of Curvelet transform are introduced and a novel image watermark scheme based on Curvelet and human visual system is proposed. At first, the scrambling digital watermark image via a layer of wavelet decomposition. Then, the original carrier image is transformed via Curvelet. At last, we embed low frequency components of watermark image into the larger 16-directional coefficient matrix of fourth layer of carrier image. Experimental results show that the proposed algorithm is simple and effective. The difference between the original image and the embedded watermark image is tiny. Simultaneously, the embedded watermarking can be extracted easily and accurately. Moreover, the algorithm has good imperceptivity and robustness for various attacks such as cropping, noise adding, and rotating and altering.

\section{Curvelet Transform}

2.1. The Theory of Curvelet Transform. Wavelet theory emerged in the mid-1980s. The good capability of time and frequency analysis is the important reason for its rapid development. However, wavelet transform cannot ideally achieve the optimal approximation order of signal for twodimensional or higher "line of singularity." So, Ridgelet theory was arisen at the historic background of theory. The basic theories of Ridgelet transform are given by Candès in 1998 [15]. It transforms "line of singularity" in the twodimensional function into "point of singularity," and then wavelet transform can obtain the optimal nonlinear approximation order for the "line of singularity" of two-dimensional or high dimensional function. Monoscale Ridgelet transform [16] and Curvelet transform [17] are developed based on the Ridgelet transform. They derive from function localization and frequency band splitting, respectively, and both can approximately represent line and curve singularity. Differently, the basic scale $s$ of monoscale Ridgelet transform is

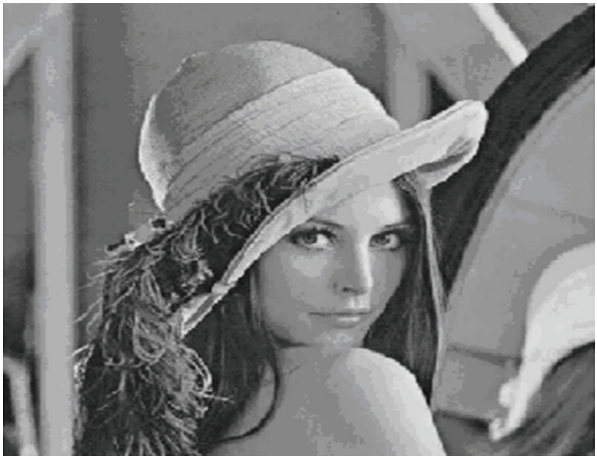

FIGURE 1: Image of Lena.

fixed; the basic scale $s$ of Curvelet transform is arbitrary and can be decomposed in any possible scale. Curvelet transform is a mixture of special filter process and multiscale Ridgelet transform as follows. Achieving Curvelet transform requires a series of filters: $\phi_{0}, \psi_{2 s}(s=0,1,2, \ldots)$. The filter maps the function $f$ as follows:

$$
\begin{aligned}
& f \longmapsto\left(P_{0} f=\phi_{0} * f, \Delta_{0} f=\psi_{0} * f, \Delta_{1} f=\psi_{2}\right. \\
& \left.\quad * f, \ldots, \Delta_{s} f=\psi_{2 s} * f, \ldots\right) .
\end{aligned}
$$

The coefficients of Curvelet transform are as follows:

$$
\alpha_{\mu}=\left\langle\Delta_{s} f, \varphi_{\mathrm{Q}, \alpha}\right\rangle, \quad Q \in \Omega_{s}, \alpha \in \Gamma .
$$

Curvelet transform maps arbitrary mean square integrable function $f$ as transform coefficient $\alpha_{\mu}(\mu \in M)$, where the parameter set of $\alpha_{\mu}$ is $M$ and $\sigma_{\mu}=\Delta_{s} \psi_{\mathrm{Q}, \alpha}$ is called Curvelet. The set of Curvelet constitutes a compact framework of $L^{2}\left(R^{2}\right)$ and can be decomposed as

$$
f=\sum_{\mu \in M}\left\langle f, \sigma_{\mu}\right\rangle \sigma_{\mu}
$$

Support interval of Curvelet bases is the most core relationship of Curvelet transform and satisfies the following:

$$
\text { width } \propto \sim \text { length }{ }^{2} \text {. }
$$

It denotes anisotropy scaling relationship. Curvelet is a kind of directional basis atom and Curvelet transform is a kind of multidirectional, multiresolution, and band-pass function analysis method. USFFT and WRAP algorithm for Curvelet transform are presented by Candès [18].

2.2. Analysis of Curvelet Coefficients Characteristics. The coefficients of $C\{j\}\{l\}\left(k_{1}, k_{2}\right)$ structure can be obtained via Curvelet transform, where $j, l$, and $\left(k_{1}, k_{2}\right)$ denote scale, direction, and $l$ th direction matrix coordinate in scale layer $j$, respectively. Lena image (Figure 1) with the size of $512 \times$ 512, for example, is divided into six-scale layer via Curvelet transform. Its innermost layer, namely, the first layer, is called Course scale layer, which is a matrix made up of low frequency coefficient and occupies the vast majority of energy of the coefficients, including the general picture of 
TABLE 1: The coefficient structure of Curvelet transformation.

\begin{tabular}{|c|c|c|c|c|c|c|}
\hline Layer & Scale coefficient & $\begin{array}{l}\text { Number of } \\
\text { directional } \\
\text { parameters }\end{array}$ & \multicolumn{4}{|c|}{ Matrix form } \\
\hline Course & $C\{1\}$ & 1 & \multicolumn{4}{|c|}{$32 \times 32$} \\
\hline \multirow{4}{*}{ Detail } & $C\{2\}$ & $32(4 \times 8)$ & $16 \times 12$ & $12 \times 16$ & $16 \times 12$ & $12 \times 16$ \\
\hline & $C\{3\}$ & $32(4 \times 8)$ & $32 \times 22$ & $22 \times 32$ & $32 \times 22$ & $22 \times 32$ \\
\hline & $C\{4\}$ & $64(4 \times 16)$ & $64 \times 22$ & $22 \times 64$ & $64 \times 22$ & $22 \times 64$ \\
\hline & $C\{5\}$ & $64(4 \times 16)$ & $128 \times 44$ & $44 \times 128$ & $128 \times 44$ & $44 \times 128$ \\
\hline Fine & $C\{6\}$ & 1 & \multicolumn{4}{|c|}{$512 \times 512$} \\
\hline
\end{tabular}

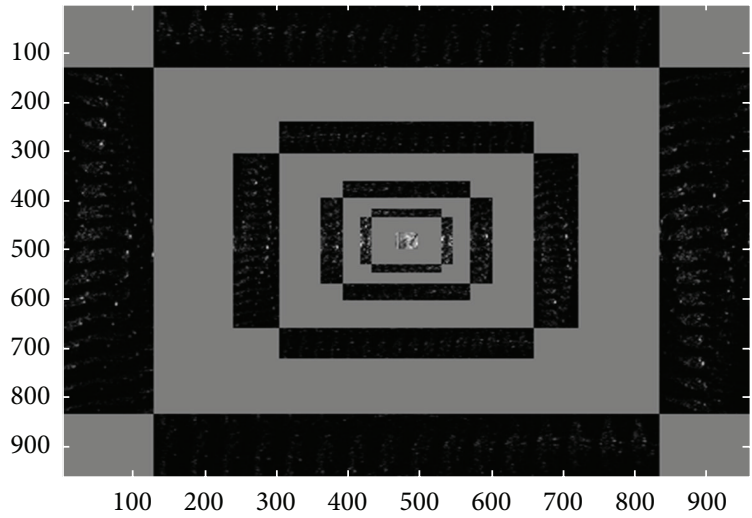

FIgURE 2: The coefficient matrix image of the first five layers via Curvelet transform.

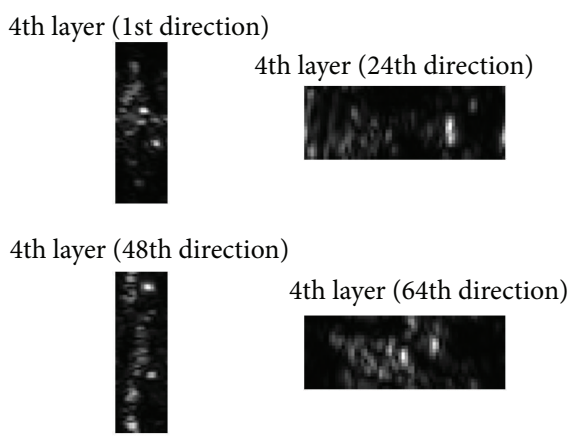

FIGURE 3: The 4th coefficient matrix image in different directions of Lena image via Curvelet transform.

image. The outermost layer, namely, the 6th layer, is called fine scale layer, which is a matrix composed of high frequency coefficient, and shows image edges and details. The 2 5 layer in the middle is called the detail scale layers. Coefficient of each layer is stratified into four broad directions and each broad direction is divided into 8, 8, 16, and 16 small directions. Each small direction is a matrix composed of medium and high frequency coefficients and the matrix is called subband coefficients matrix. Figure 2 shows coefficient matrix images of the first five layers; Figure 3 shows the coefficient matrix image of different directions in the 4 th layer. Figure 4 is the outermost layer coefficient matrix image.

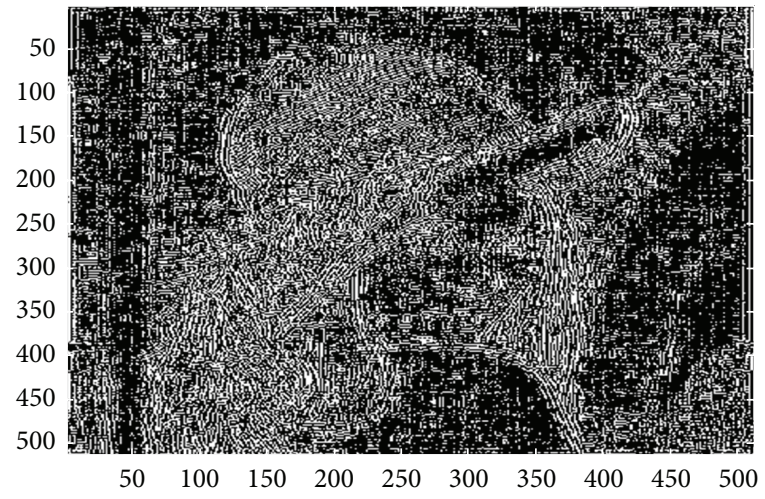

Figure 4: The coefficient matrix image in 6th layer of Lena image via Curvelet transform.

The transform coefficient format of matrix coordinate $\left(k_{1}, k_{2}\right)$ in $l$ th direction of $j$ th layer is shown in Table 1.

\section{Wavelet Transform}

Wavelet analysis, as a mathematical theory and method, was developed from the mid-1980s. It is proposed by the French scientists Grossma and Morlet during their analysis of the seismic signals [19]. Wavelet analysis was regarded as a breakthrough of Fourier analysis and has good localization performance in time-frequency domain and solved many difficult problems that cannot be solved by the Fourier transform. The wavelet transform is known as the "mathematical microscope," which is a milestone in the history of harmonic analysis development. The main idea of wavelet transform is to perform a detailed frequency separation for image, known as the multiresolution transform. Image can be stratified into the low frequency domain (on behalf of the general picture of the image) and the high frequency domain (on behalf of the image detail) via wavelet transform. And then, the high frequency part can be divided into three parts: $\mathrm{HL}, \mathrm{LH}$, and $\mathrm{HH}$, which represents horizontal, vertical, and diagonal direction of the image, respectively. The low frequency coefficient represents average brightness of the corresponding wavelet blocks and high frequency coefficient represents the image texture and edges. If low frequency region is further subdivided via wavelet transform, it will generate a higher order of low frequency and higher 


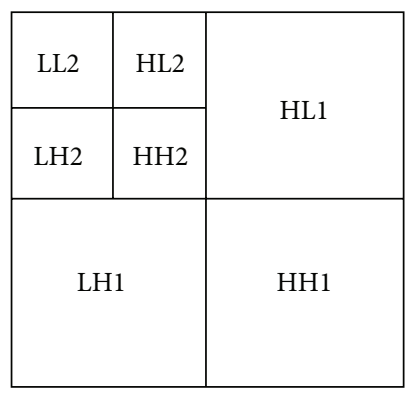

Figure 5: The structure diagram of the two-order wavelet decomposition.

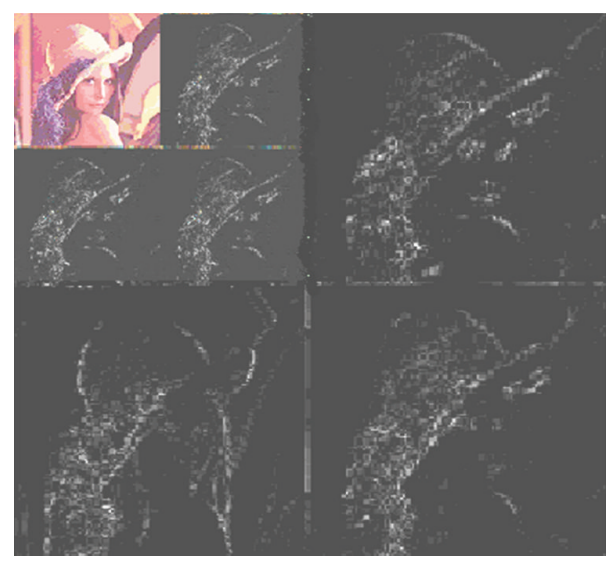

FIGURE 6: Lena image via two-order wavelet decomposition.

frequency order in accordance with the above recursion principle. Figure 5 denotes the structure diagram of the twoorder wavelet decomposition and Figure 6 shows the colored Lena image via two-order wavelet decomposition.

\section{Watermark Algorithm and Evaluation Criterion}

4.1. Image Scrambling Transform. Image scrambling transform which is an algorithm via messing image pixel sequence is a kind of image encryption technology. The number of pixels and the histogram of the image are not changed. The scrambling watermark can eliminate the spatial interdependency of watermark pixels. So it enhances the robustness of watermark image for clipping operation.

The scrambling methods, such as Fass curve, Graycode, Arnold transform, and magic square, have been used. According to intuitionism and periodicity of the Arnold transform, we preprocessed the watermark image via the transform [20]. Arnold transform, known as Arnold's Cat Map, is an ergodic theory. From the view of the theory of sampling, digital image is a two-dimensional discrete sampling array, namely, an image matrix. Discrete Arnold transform can be operated for a square digital image:

$$
\left(\begin{array}{l}
x^{\prime} \\
y^{\prime}
\end{array}\right)=\left[\left(\begin{array}{ll}
1 & 1 \\
1 & 2
\end{array}\right)\left(\begin{array}{l}
x \\
y
\end{array}\right)\right] \bmod N,
$$

where $(x, y)$ and $\left(x^{\prime}, y^{\prime}\right)$ denote the anterior pixel location and posterior pixel location via Arnold transform, respectively. The scrambling image can be produced via Arnold transform.

4.2. Watermark Algorithm and Evaluation Criterion. Lena image $(512 \times 512)$ is executed Curvelet transform via USFFT. According to the structure of the human eye and knowledge of human visual system, human eyes are more sensitive to low frequency signal than to high frequency information. Taking into account robustness and imperceptibility of watermark information, watermark information is embedded into the detail scale level. The first 16-direction subbands with higher energy are chosen for watermark embedded site. Concrete steps of embedding watermark information are as follows.

Step 1. Execute Curvelet decomposition on Lena image.

Step 2. Turn each of the coefficient matrixes of 64 small directions in the 4th layer into $1 * 1408$ row vectors and compute the energy value in each direction. The formula is as follows:

$$
E_{4}^{(i)}=\sum_{n=1}^{1408}\left|d_{4, i}^{(2)}(1, n)\right|^{2}, \quad i=1,2, \ldots, 64 .
$$

Step 3. Select the first 16 directions with larger energy as the watermark carrier site.

Step 4. Execute Arnold scrambling for original watermark $(64 * 64)$ and then via wavelet transform (Figure 7$)$.

Step 5 . Turn the low frequency matrix $(32 * 32)$ into $1 * 1024$ row vectors, which is used as watermark information.

Step 6. Embed watermark information into the first 16 directions with larger energy vector coefficient in Step 3, and change them into direction matrix respectively:

$$
d_{4, i_{l}}^{w}(1, j)=d_{4, i_{l}}(1, j)+\alpha w_{j}, \quad j=1,2, \ldots, 1024 .
$$

Step 7. Execute Curvelet reconstruction to obtain the embedded watermark image (Figure 8).

The extraction of the watermark is implemented via inverse process of the watermark embedding; specific steps are as follows.

Step 1. Execute Curvelet decomposition for Lena image which have been embedded with watermark information.

Step 2. Turn the coefficient matrix of 64 small directions in the fourth layer into $1 * 1408$ row vectors, respectively. Then subtract coefficient matrix row vector corresponding to 

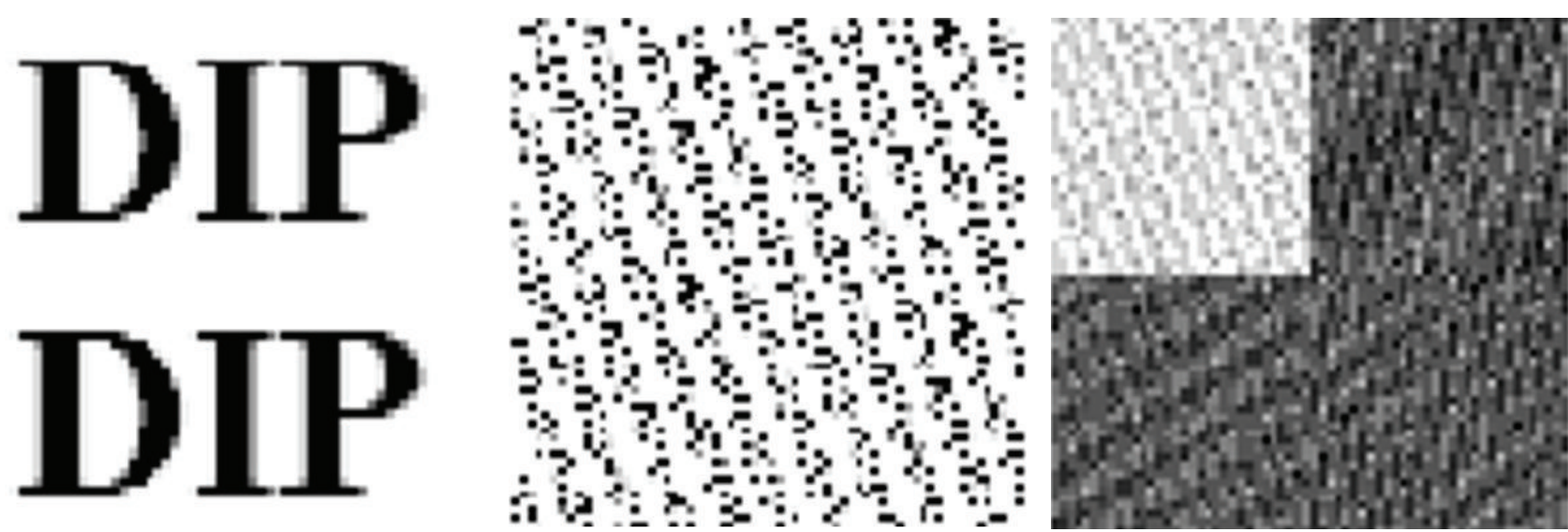

FIGURE 7: The original watermark image, Arnold scrambling image, and wavelet decomposition image.
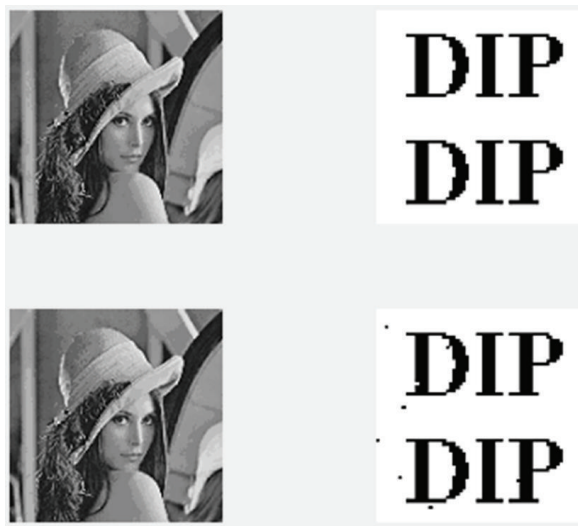

FIGURE 8: The original image, the original watermarking, embedded watermark image, and the extracted watermarking.

original Lena image and multiply the reciprocal of 16 times embedding strength factor:

$$
\begin{aligned}
W_{1, j}=\frac{1}{16 \alpha}\left[d_{4, i_{l}}^{w}(1, j)-d_{4, i_{l}}(1, j)\right], & \\
& j=1,2, \ldots, 1408 .
\end{aligned}
$$

Step 3. Turn the first 1024 elements of vector $W_{1, j}$ into the $32 *$ 32 matrix, which is extracted watermark information.

Step 4. Combine extracted watermark information with high frequency component obtained by a layer of wavelet decomposition of the original watermark and make a wavelet reconstruction.

Step 5. Execute inverse Arnold scrambling for the reconstructed image and watermark image is thus extracted (Figure 8).

The human eye which acts as the digital image processing terminal is the most accurate method for the subjective evaluation. However, subjective evaluation methods are all complex, time-consuming, and difficult to quantify. So the limitations of subjective evaluation promote the development of objective evaluation method. The traditional objective evaluation methods such as Peak Signal to Noise Ratio (PSNR) and Mean Square Estimation (MSE) are widely used in image quality evaluation [21]. However, they are not combined with the human visual system and cannot accurately measure the watermarked image visual quality. Therefore, it is necessary to seek a more objective and accurate evaluation. Recently, the structural similarity index (SSIM) which acts as a novel objective evaluation of two-image similarity index is gradually applied [22]. It comes from the improvement of the universal image quality. Structural similarity of images consists of comparison of brightness, contrast, and structure information; their expression is as follows:

$$
\begin{aligned}
& l(x, y)=\frac{2 u_{x} u_{y}+C_{1}}{u_{x}^{2}+u_{y}^{2}+C_{1}}, \\
& c(x, y)=\frac{2 \sigma_{x} \sigma_{y}+C_{2}}{\sigma_{x}^{2}+\sigma_{y}^{2}+C_{2}}, \\
& s(x, y)=\frac{2 \sigma_{x y}+C_{3}}{\sigma_{x} \sigma_{y}+C_{3}} .
\end{aligned}
$$

They form evaluation method:

$$
\operatorname{SSIM}(x, y)=[l(x, y)]^{\alpha}[c(x, y)]^{\beta}[s(x, y)]^{\gamma},
$$

where $x, y, u_{x}, u_{y}$ represent, respectively, the original image blocks and the degraded image blocks and denote, respectively, their corresponding variance and are all small positive constants and $\sigma_{x y}$ is covariance of $x$ and $y$. In order to adjust the proportion of three parts, given parameters $\alpha, \beta, \gamma$ are greater than zero and $C_{1}, C_{2}, C_{3}$ are all small constant to avoid the denominator is zero, where $C_{1}=\left(K_{1} L\right)^{2}, C_{2}=$ $\left(K_{2} L\right)^{2}, C_{3}=\left(K_{3} L\right)^{2}, C_{3}=C_{2} / 2$, and $L$ is the dynamic range of pixel values. SSIM utilizes image information in different frequency bands and different directions and also 

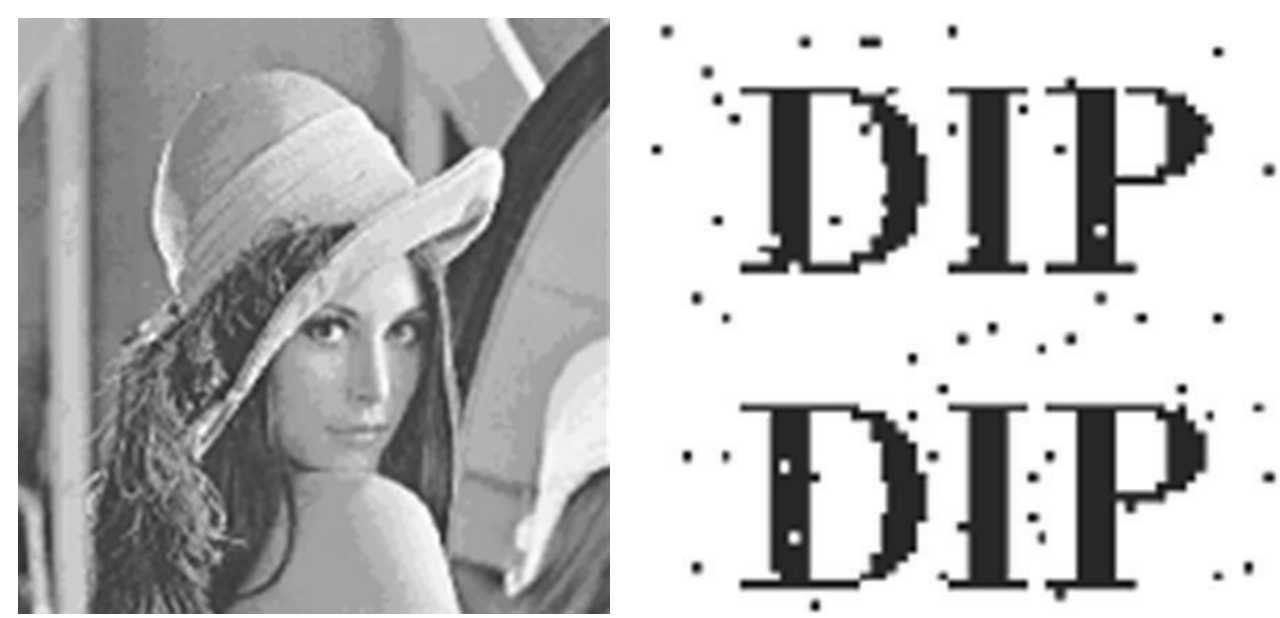

FIGURE 9: Uniform random noise attack image and extracted watermark $(\mu=0, \delta=1)$.

can capture local statistical characteristics of the image. Compared with PSNR, it is more consistent with human visual system and accords with the objective evaluation of the embedded watermark image. To value the image quality, the image is stratified into several overlapping or nonoverlapping subblocks and each subblock SSIM is calculated; then average summation of subblock SSIM and obtain the whole image MSSIM, namely,

$$
\operatorname{MSSIM}(X, Y)=\frac{1}{M} \sum_{i=1}^{M} \operatorname{SSIM}\left(x_{i}, y_{i}\right),
$$

where $X, Y$ represents the original image block and degraded image blocks, respectively, $M$ is the total account of image subblocks, and $x_{i}, y_{i}$ denotes the $i$ th subblock of the original image and the distortion image, respectively. In this paper, MSSIM index is an objective measure of the hidden watermark. To quantify consequent, using more concrete form of MSSIM index,

$$
\operatorname{MSsim}(x, y)=\frac{\left(2 \mu_{x} \mu_{y}+C_{1}\right)\left(2 \sigma_{x y}+C_{2}\right)}{\left(\mu_{x}^{2}+\mu_{y}^{2}+C_{1}\right)\left(\sigma_{x}^{2}+\sigma_{y}^{2}+C_{2}\right)}
$$

In order to eliminate the effect of subjective and objective factors such as experience, physical condition of the observers, and experimental conditions, Normalized CrossCorrelation (NC) is adopted to quantitatively evaluate the similarities between extracted watermark $\widetilde{W}$ and the original watermark $W[23]$, which is defined as follows:

$$
\begin{aligned}
& \mathrm{NC}(W, \widetilde{W}) \\
&=\frac{\sum_{i=1}^{M} \sum_{j=1}^{N} w(i, j) \cdot \widetilde{w}(i, j)}{\sqrt{\sum_{i=1}^{M} \sum_{j=1}^{N} w^{2}(i, j)} \cdot \sqrt{\sum_{i=1}^{M} \sum_{j=1}^{N} \widetilde{w}^{2}(i, j)}} .
\end{aligned}
$$

Generally, if the extracted watermark $\widetilde{W}$ and the original watermark $W$ satisfy the relationship $\operatorname{NC}(W, \widetilde{W})>0.85$, it is shown that the extracted watermark has good similarity with original one and the watermarking satisfies the requirements of robustness. The greater the relative value, the better the robustness.

4.3. Watermark Attack Analysis. In order to detect the imperceptibility of watermark information and robustness of the algorithm, cutting edge attack, Gaussian noise attack, a quarter of the clipping attack, and rotating and altering attack are simulated to test the Lena image. All kinds of attack effects are shown in Figures 9-24.

As can be seen from Table 2, the embedded watermark image has very good imperceptibility and can resist various attacks. Moreover, the extracted watermark image and the original one have much correlation, satisfying the requirement of the watermark robustness. Furthermore, the proposed algorithm in the paper is better robustness to the rotational attack than other transforms; Table 3 is the test results of the spinning processing attack $\left(\theta=30^{\circ}\right)$ for various transforms.

\section{Conclusions}

In this paper, the basic principles of Curvelet transform have been analyzed. Compared with wavelet transform and Ridgelet transform, Curvelet transform shows better performance in signal processing. We take full advantage of multidirection, multiresolution, and band-pass features of Curvelet transform and the characteristics of human visual system. The scrambling digital watermark information is embedded into the first 16 directions with larger energy in the fourth layer. All kinds of attack are simulated on embedded watermark image. Experimental results show that the algorithm has good robustness and imperceptibility and better robustness to the spinning processing attack than other transforms. In the future, we will do a further study on the 

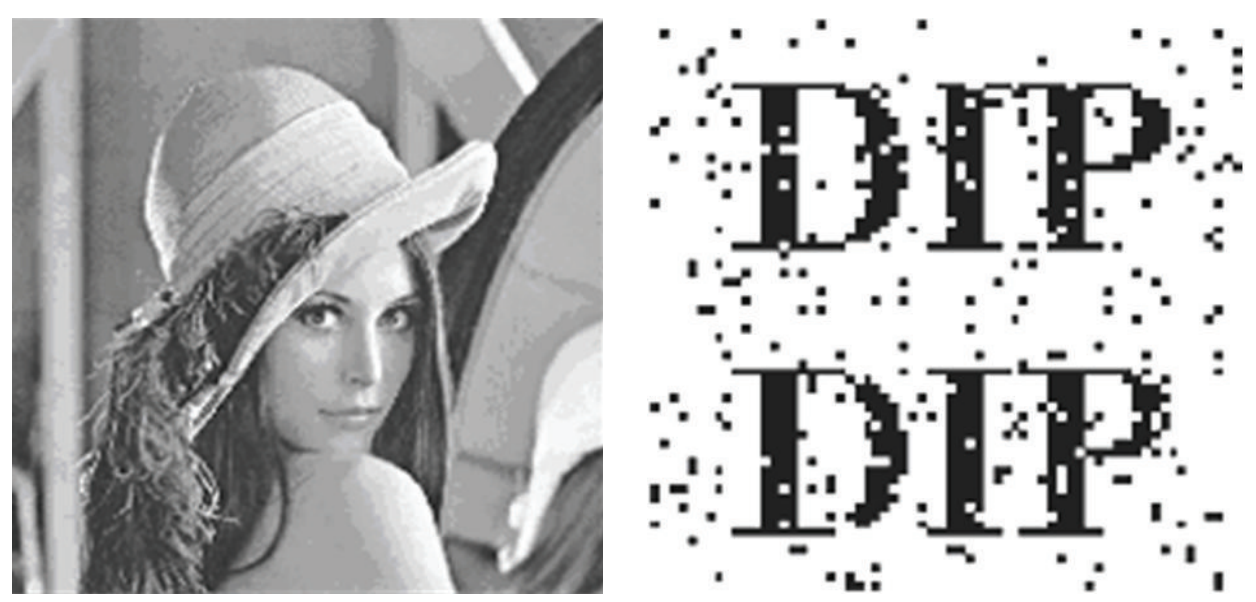

FIGURE 10: Uniform random noise attack image and extracted watermark $(\mu=0, \delta=4)$.
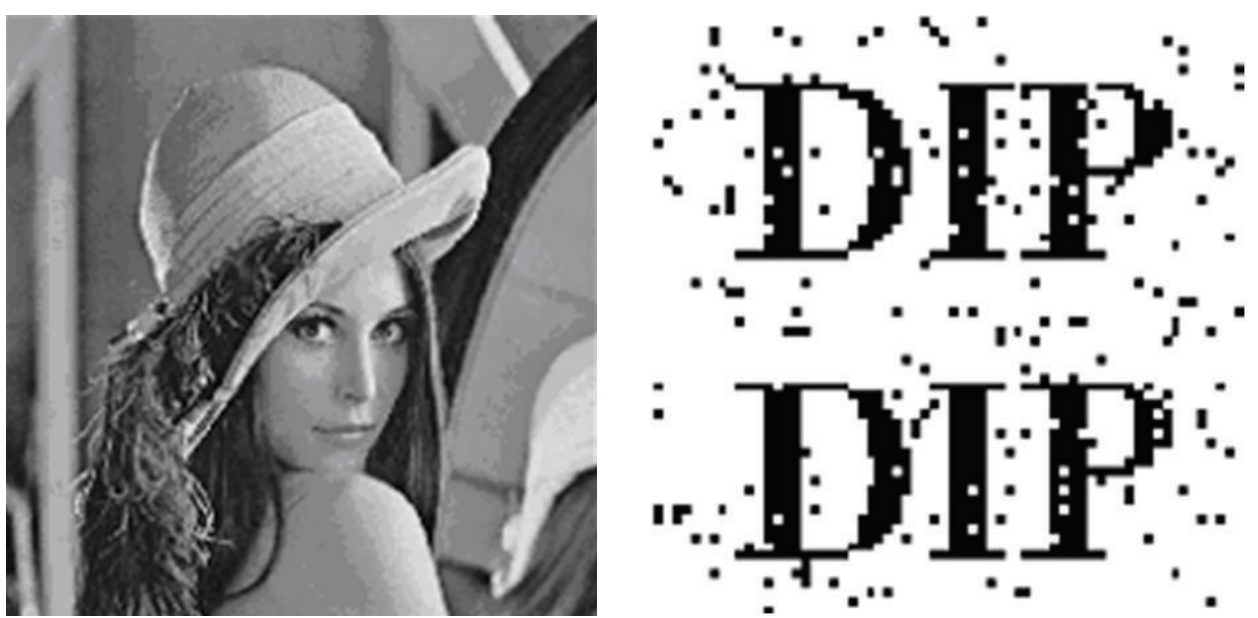

FIGURE 11: Normal random noise attack image and extracted image $(\mu=0, \delta=1)$.
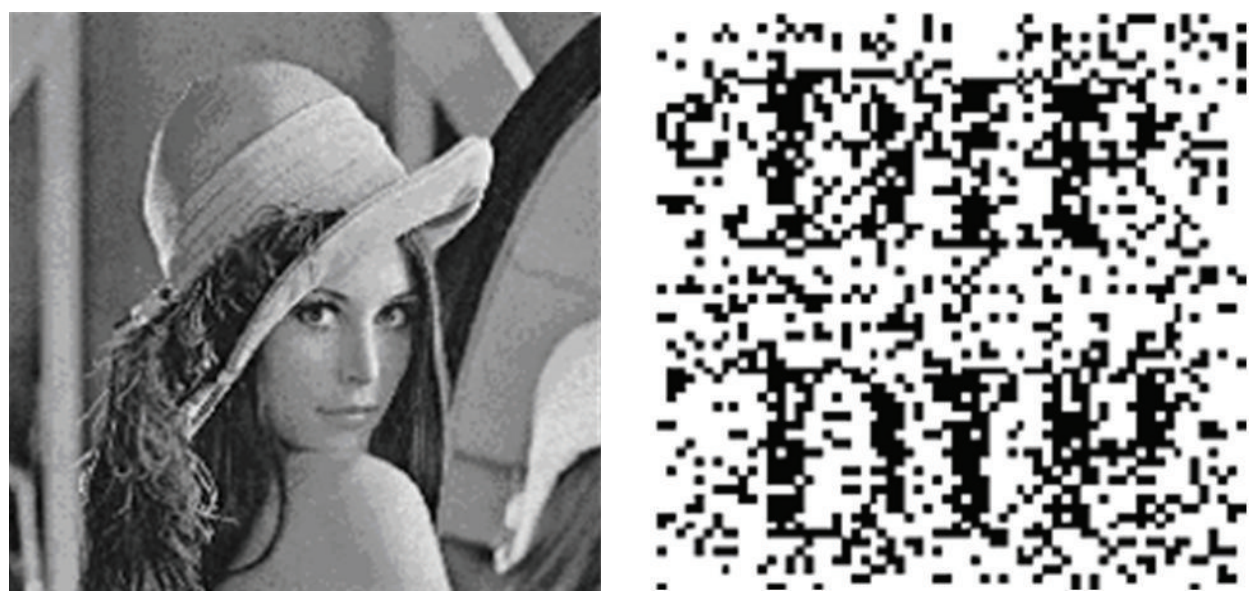

FIGURE 12: Normal random noise attack image and extracted image $(\mu=0, \delta=4)$. 

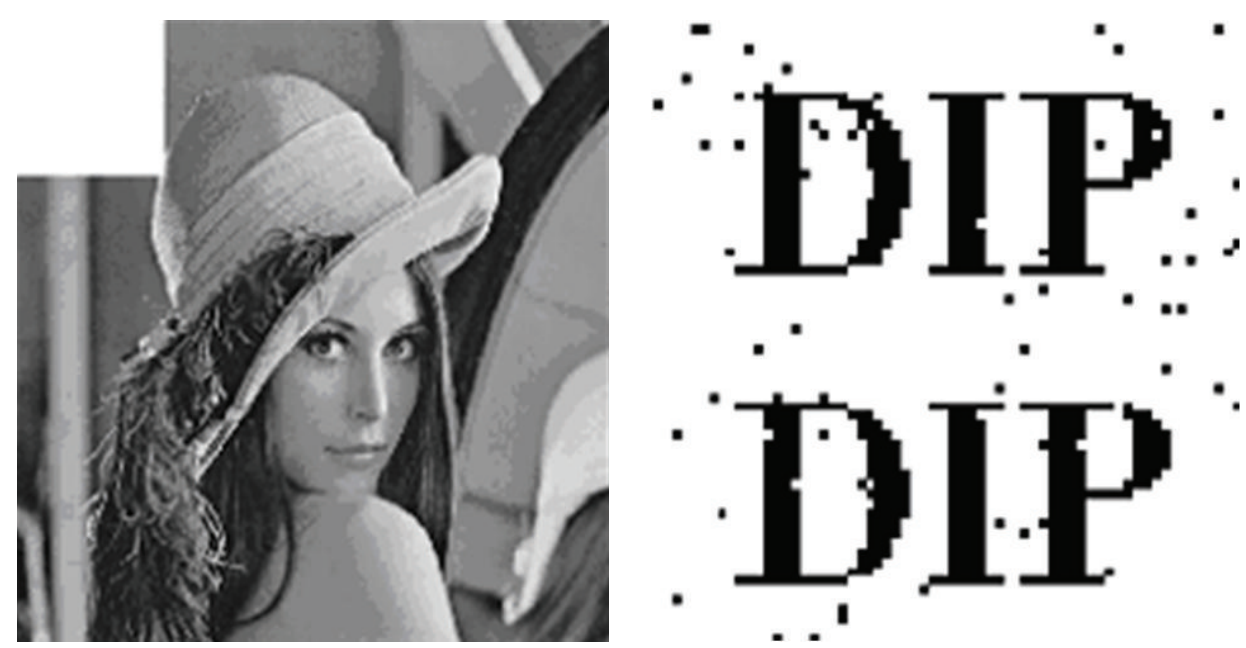

FIGURE 13: Cropping attack image and extracted image (1/16).
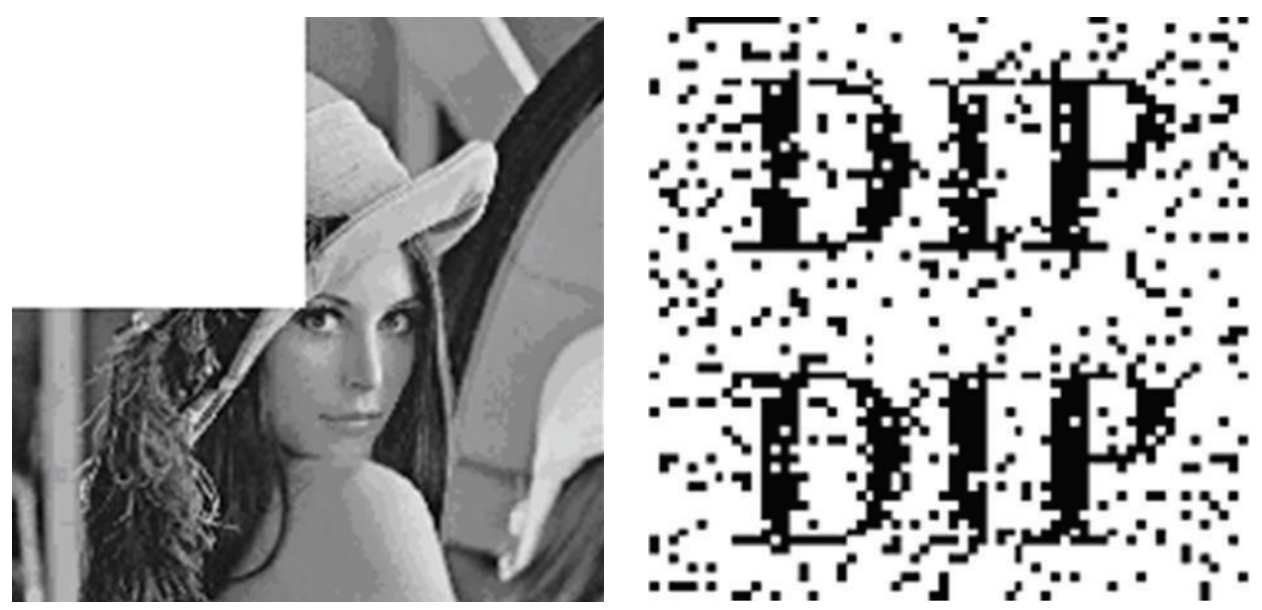

FIGURE 14: Cropping attack image and extracted image (1/4).
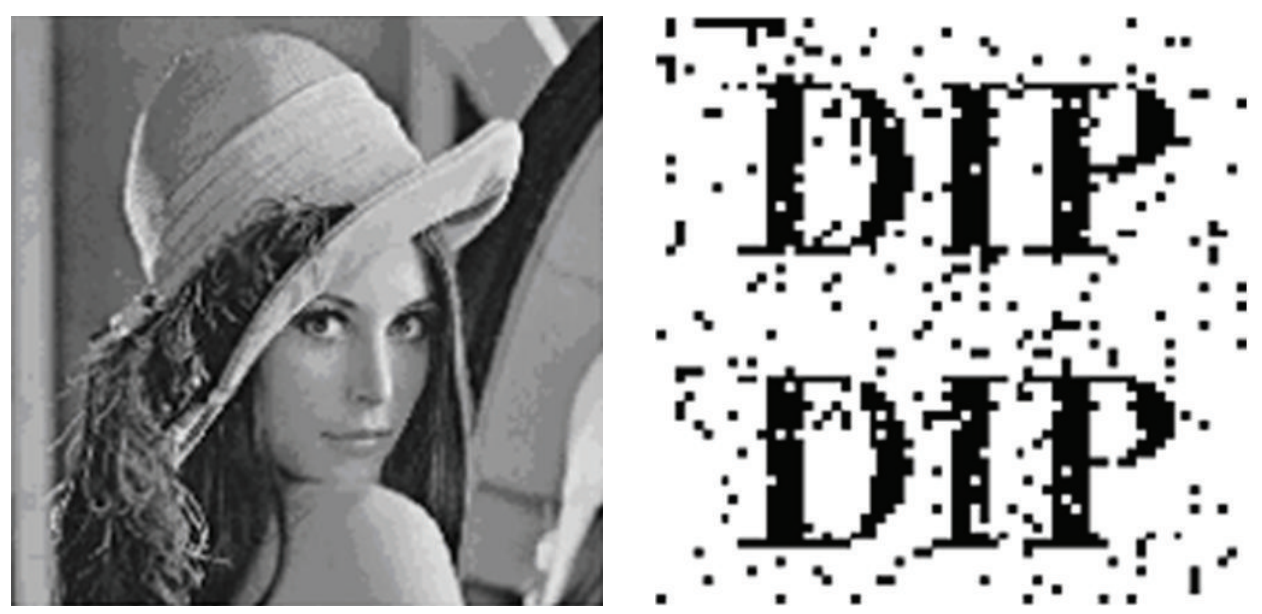

FIgURE 15: Cropping edges attack image and extracted image. 

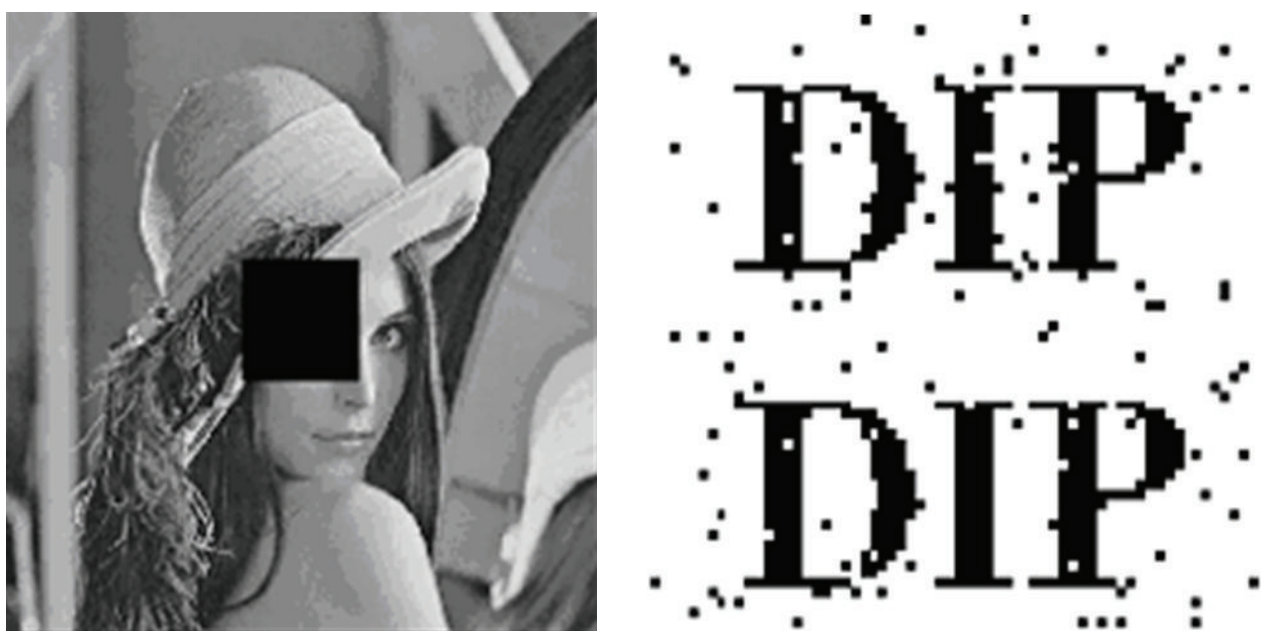

FIGURE 16: Graffiti attack image and extracted image.
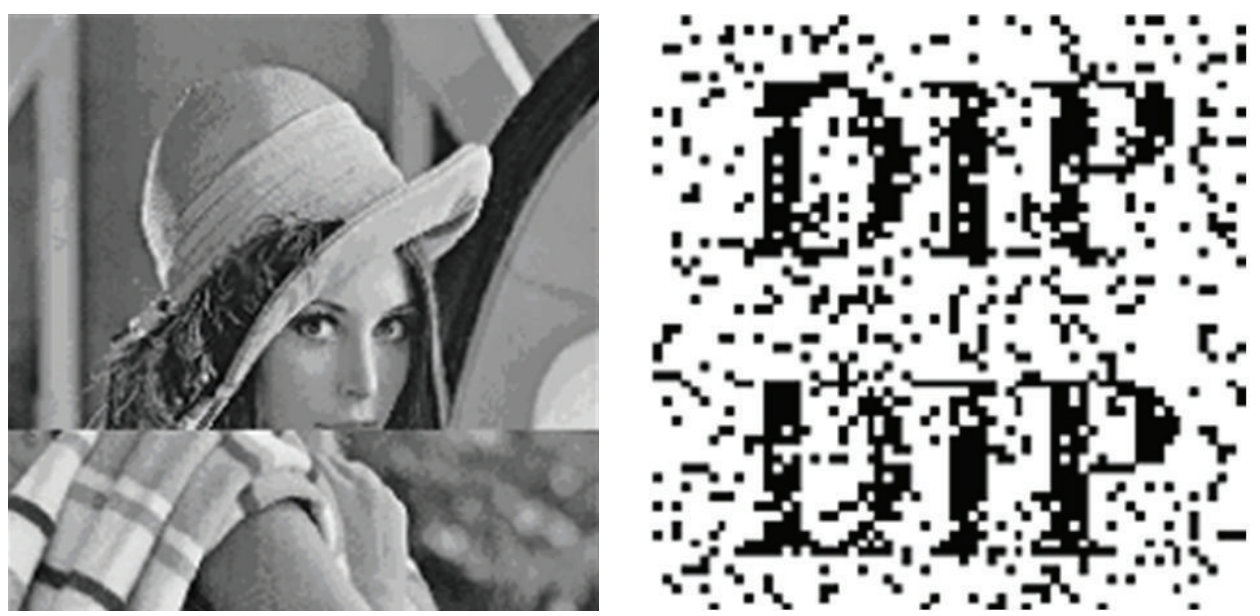

FIGURE 17: Montage attack image and extracted image.
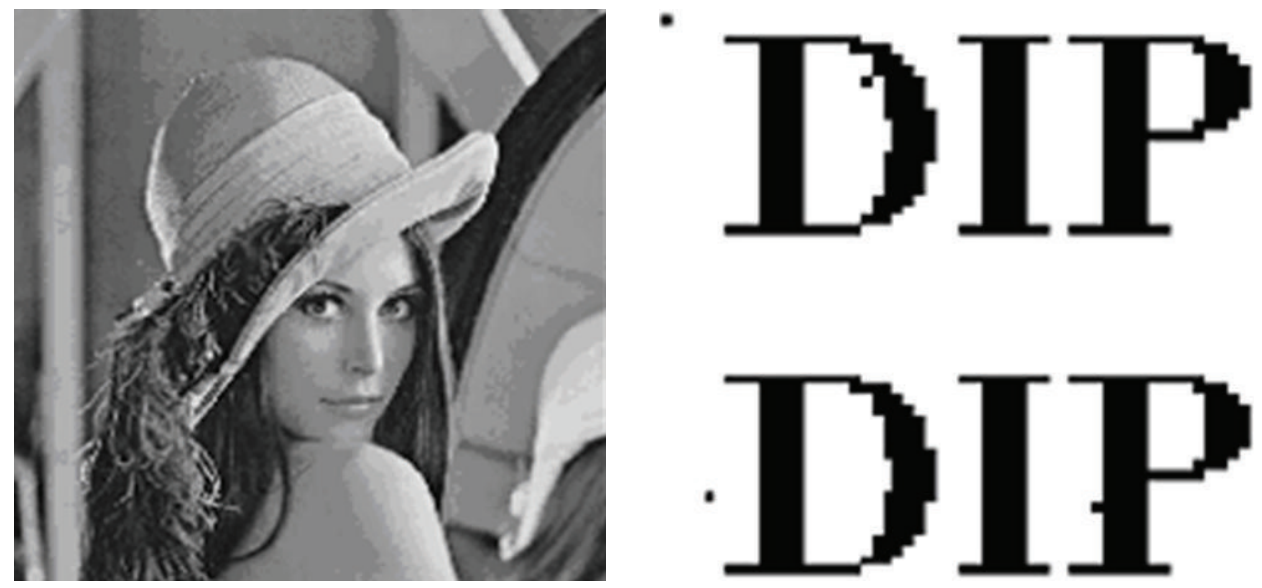

FIGURE 18: Half brightness attack image and extracted image. 

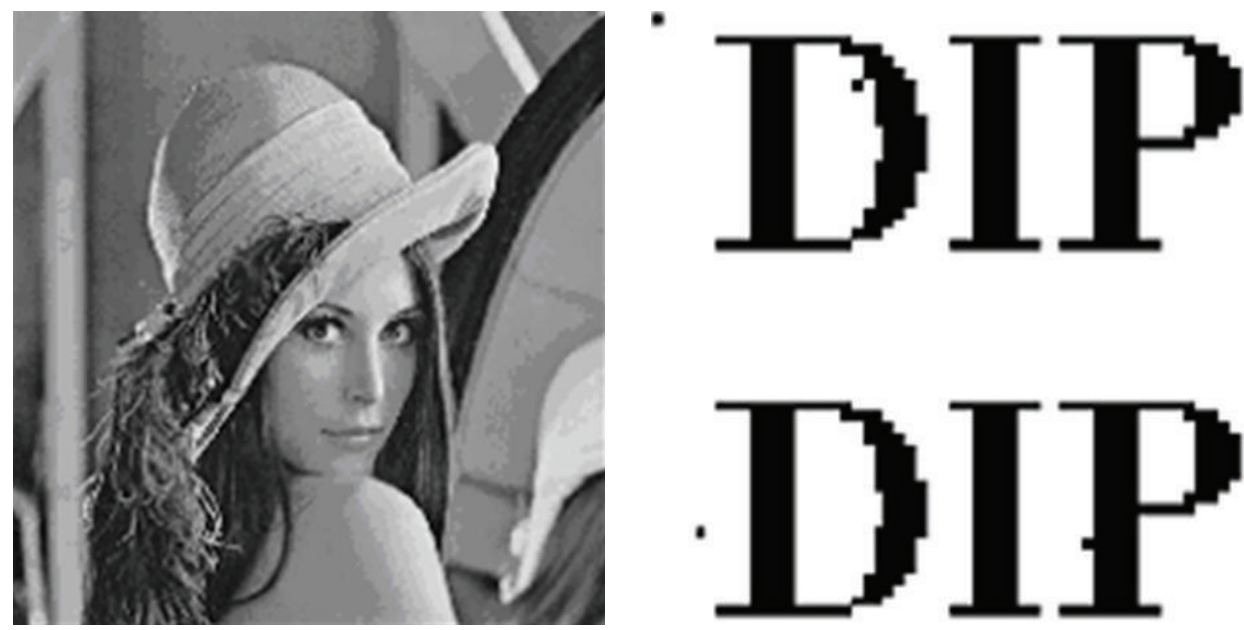

FIGURE 19: Double brightness attack image and extracted image.
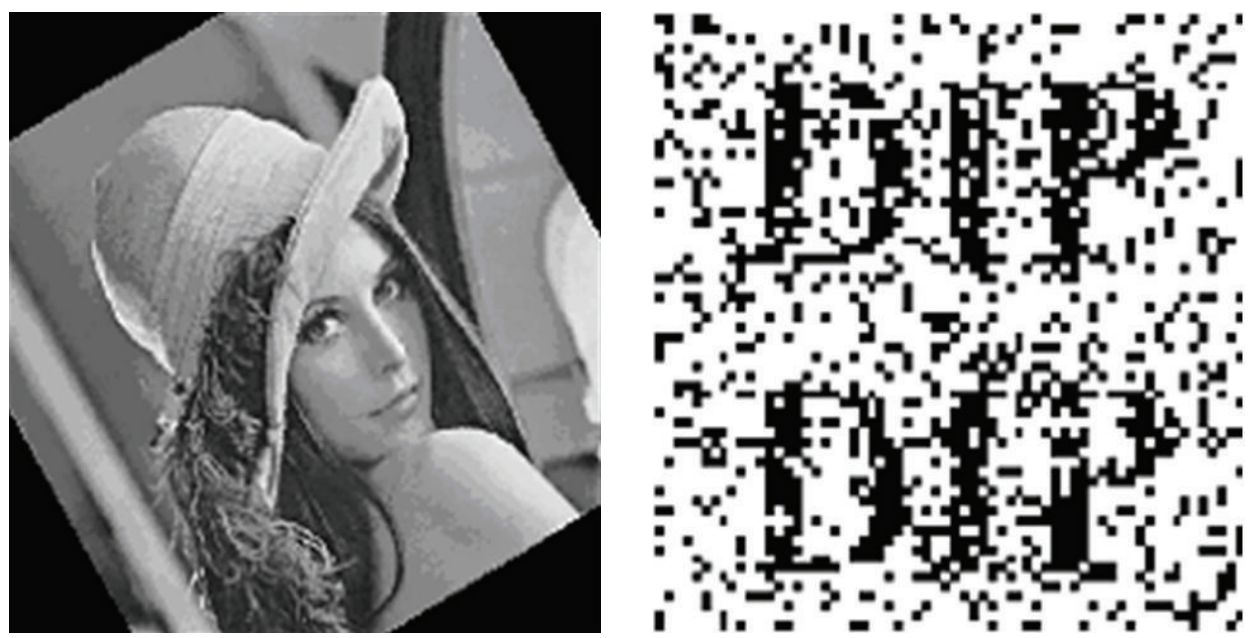

FIgURE 20: Rotation attack image and extracted image $\left(\theta=30^{\circ}\right)$.
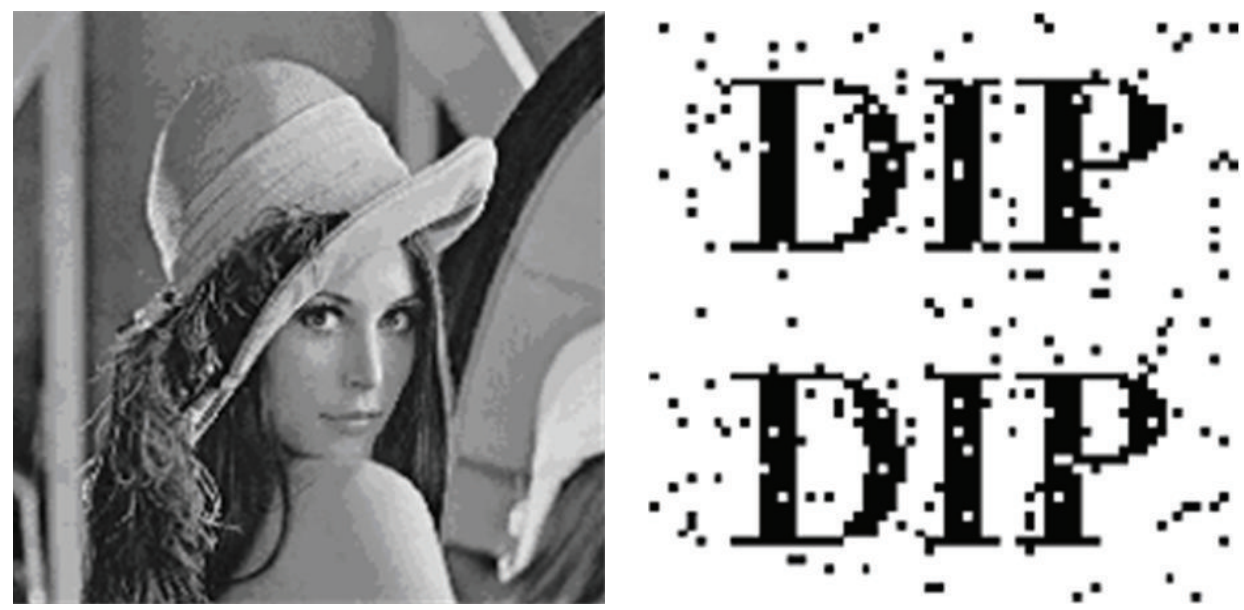

FIGURE 21: Compression attack image and extracted image (85\%). 

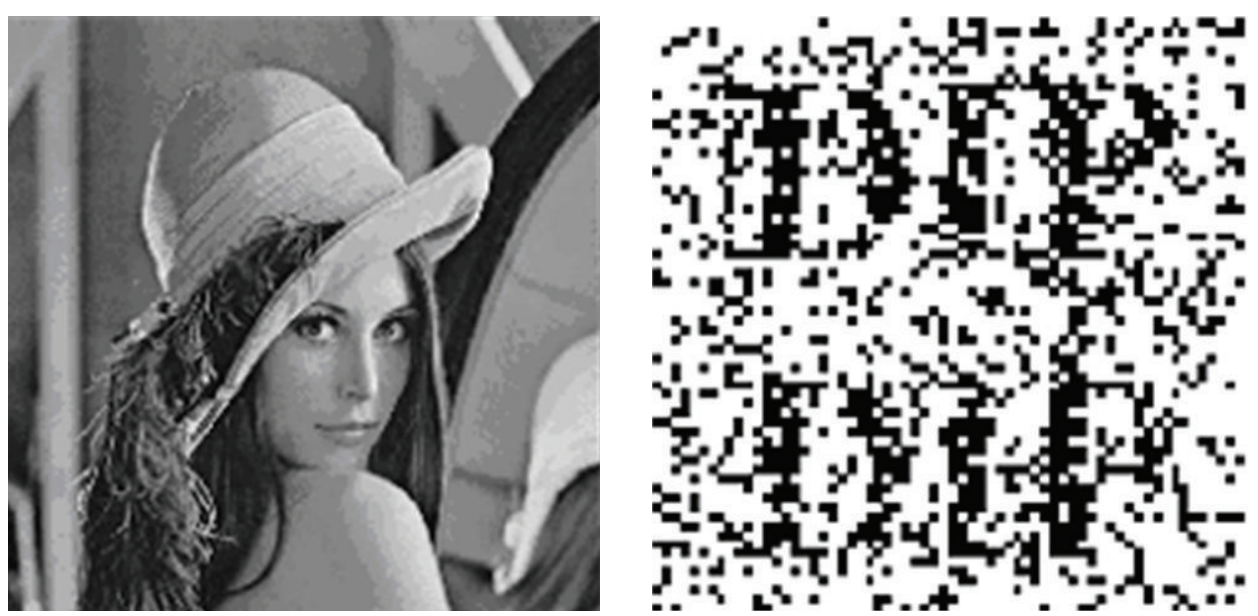

FIGURE 22: Compression attack image and extracted image (70\%).
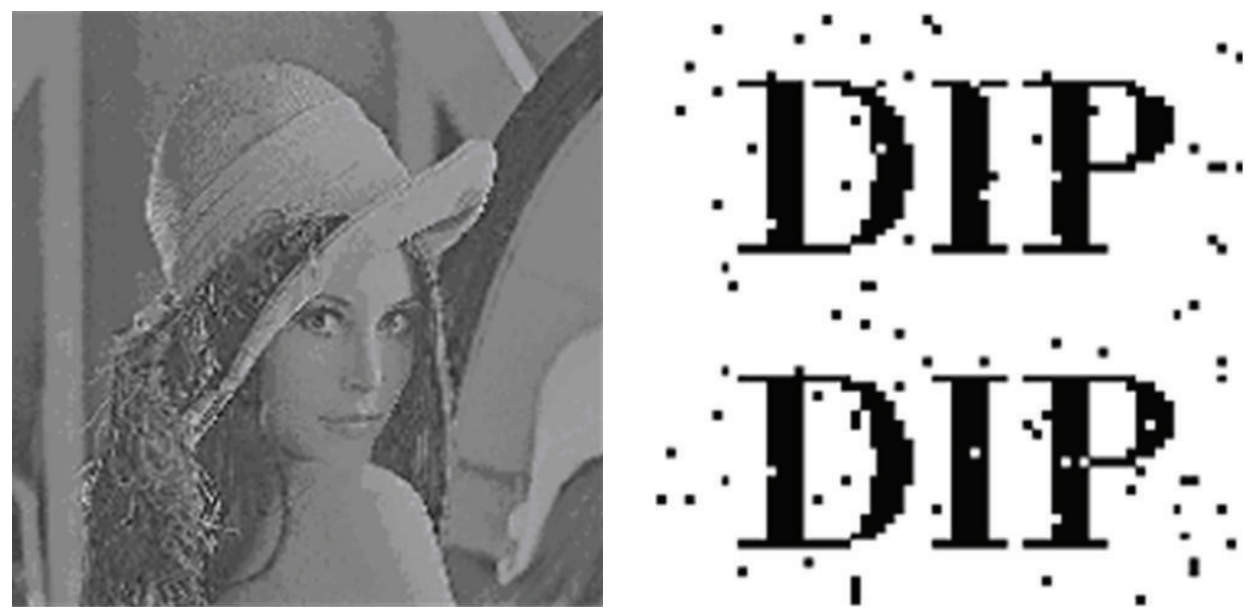

FIGURE 23: Sharp filtering attack image and extracted image.
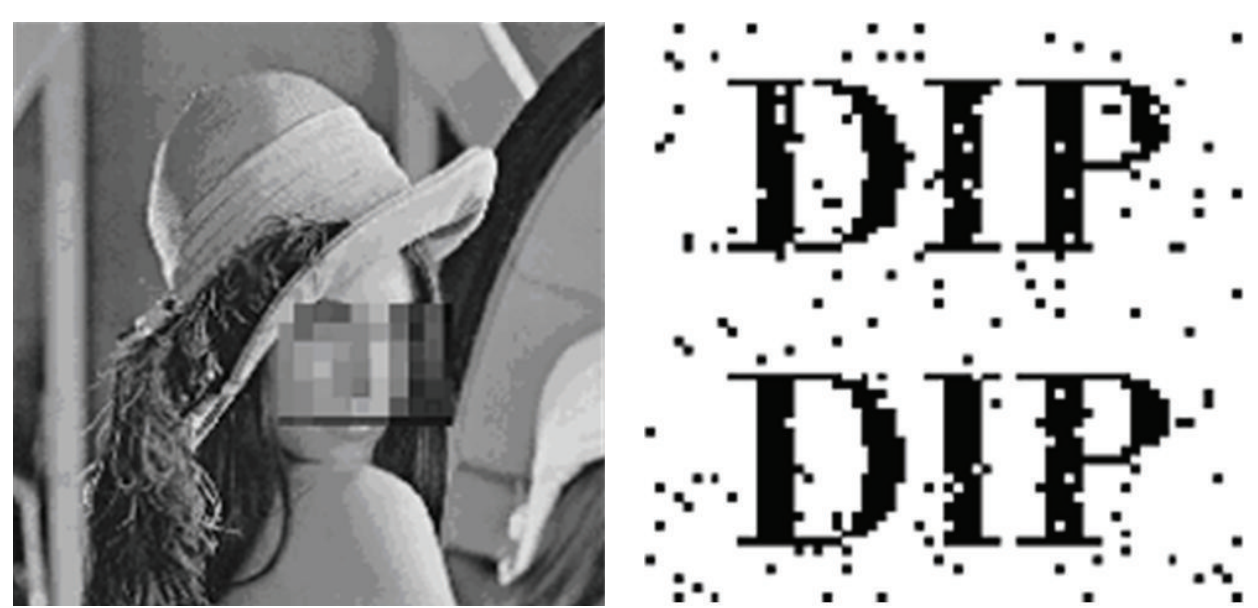

Figure 24: Mosaic attack image and extracted image. 
TABLE 2: Watermark algorithm test results against different attacks.

\begin{tabular}{lcccccccc}
\hline $\begin{array}{l}\text { Attack } \\
\text { mode }\end{array}$ & No attack & $\begin{array}{c}\text { Uniform } \\
\text { noise (1) }\end{array}$ & $\begin{array}{c}\text { Uniform } \\
\text { noise (2) }\end{array}$ & $\begin{array}{c}\text { Normal } \\
\text { noise }(1)\end{array}$ & $\begin{array}{c}\text { Normal } \\
\text { noise (2) }\end{array}$ & $\begin{array}{c}\text { Cropping } \\
(1)\end{array}$ & $\begin{array}{c}\text { Cropping } \\
(2)\end{array}$ & $\begin{array}{c}\text { Cropping } \\
\text { edges }\end{array}$ \\
\hline SSIM & 0.9998 & 0.9884 & 0.9573 & 0.9824 & 0.9499 & 0.8776 & 0.6947 & 0.8348 \\
NC & 1.0000 & 0.9837 & 0.8517 & 0.9528 & 0.7635 & 0.9822 & 0.8693 & 0.9251 \\
\hline Graffiti & Montage & $\begin{array}{c}\text { Half } \\
\text { brightness }\end{array}$ & $\begin{array}{c}\text { Double } \\
\text { brightness }\end{array}$ & Rotation & $\begin{array}{c}\text { Compress } \\
(1)\end{array}$ & $\begin{array}{c}\text { Compress } \\
(2)\end{array}$ & Sharp & Mosaic \\
\hline 0.8990 & 0.8753 & 0.8561 & 0.6431 & 0.9513 & 0.9923 & 0.9873 & 0.8867 & 0.8991 \\
0.9700 & 0.8440 & 0.9988 & 0.9988 & 0.7906 & 0.9513 & 0.7733 & 0.9768 & 0.9620 \\
\hline
\end{tabular}

TABLE 3: The test results of the spinning processing attack for various transforms.

\begin{tabular}{lcccccc}
\hline Various transforms & DFT & DCT & DWT & Ridgelet & Curvelet & Contourlet \\
\hline NC & 0.3343 & 0.6154 & 0.6455 & 0.7537 & $\mathbf{0 . 7 9 0 6}$ & 0.2415 \\
\hline
\end{tabular}

effect of the watermark which is embedded into the other scale layers.

\section{Conflict of Interests}

The authors declare that there is no conflict of interests regarding the publication of this paper.

\section{Acknowledgments}

The work was supported by the National Nature Science Foundation of China (no. 61071188) and the Nature Science Foundation of Hubei Province (no. B2015095) and the innovative personnel Foundation of Hubei Polytechnic University (no. 14xjz02C).

\section{References}

[1] F. Mintzer, W. Gordon, and M. Minerva, "Effective and ineffective digital watermarks," in Proceedings of the IEEE International Conference on Image Processing, vol. 10, pp. 343-351, Santa Barbara, Calif, USA, October 1997.

[2] M. Minerva and C. Mintzer, "Digital watermarking for highquality imaging," in Proceedings of the 1st Signal Processing Society Workshop on Multimedia, vol. 6, pp. 357-362, Princeton, NJ, USA, 1997.

[3] R. B. Wolfgang, C. I. Podilchuk, and E. J. Delp, "Perceptual watermarks for digital images and video," Proceedings of the IEEE, vol. 87, no. 7, pp. 1108-1126, 1999.

[4] I. J. Cox, L. Matt, and A. J. Bloom, Digital Watermarking, Electronic Industry Press, Beijing, China, 2003.

[5] L. K. Jones, "A simple lemma on greedy approximation in hilbert space and convergence rates for projection pursuit regression and neural network training," The Annals of Statistics, vol. 20, no. 1, pp. 608-613, 1992.

[6] R. R. Coifman and M. V. Wickerhauser, "Entropy-based algorithms for best basis selection," IEEE Transactions on Information Theory, vol. 38, no. 2, pp. 713-718, 1992.

[7] T. L. Ruanaidh and W. Sletan, "An algorithm of watermark based on discrete Fourier transform," IEEE Transactions on Image Processing, vol. 6, no. 8, pp. 663-672, 1997.
[8] J. R. Hernández, M. Amado, and F. Pérez-González, "DCTdomain watermarking techniques for still images: detector performance analysis and a new structure," IEEE Transactions on Image Processing, vol. 9, no. 1, pp. 55-68, 2000.

[9] F. Davoine, "Comparison of two wavelet based image watermarking schemes," in Proceedings of the International Conference on Image Processing (ICIP '00), pp. 682-685, Vancouver, Canada, September 2000.

[10] E. J. Candes, R. R. Coifman, and D. L. Donoho, "Digital implementation of ridgelet packets," Tech. Rep., Stanford University, Stanford, Calif, USA, 2002.

[11] E. Candès, L. Demanet, D. Donoho, and L. Ying, "Fast discrete curvelet transforms," Multiscale Modeling and Simulation, vol. 5, no. 3, pp. 861-899, 2006.

[12] W. Bender, D. Gruhl, N. Morimoto, and A. Lu, "Techniques for data hiding," IBM Systems Journal, vol. 35, no. 3-4, pp. 313-336, 1996.

[13] F. Bartolini, M. Barni, V. Cappellini, and A. Piva, "Mask building for perceptually hiding frequency embedded watermarks," in Proceedings of the International Conference on Image Processing (ICIP '98), pp. 450-454, October 1998.

[14] M. Kutter and S. Winkler, "A vision-based masking model for spread-spectrum image watermarking," IEEE Transactions on Image Processing, vol. 11, no. 1, pp. 16-25, 2002.

[15] E. J. Candes, Ridgelets: theory and applications [Ph.D. thesis], Department of Statistics, Stanford University, Stanford, Calif, USA, 1998.

[16] E. J. Candes, "Monoscale Ridgelet for the representation of images with edges," Tech. Rep., Deperment of Statistics, Stanford University, Stanford, Calif, USA, 1999.

[17] D. L. Donoho and M. R. Duncan, "Digital curvelet transform: strategy, implementation, and experiments," in Wavelet Applications VII, vol. 4056 of Proceedings of SPIE, pp. 12-29, Orlando, Fla, USA, April 2000.

[18] E. J. Candès and D. L. Donoho, "New tight frames of curvelets and optimal representations of objects with piecewise $C^{2}$ singularities," Communications on Pure and Applied Mathematics, vol. 57, no. 2, pp. 219-266, 2004.

[19] A. Grossman and J. Morlet, "Decoposition of Hardy functions into square integral wavelets of constant shape," Signal Processing, vol. 52, no. 3, pp. 375-383, 1984.

[20] T. Kong and T. Zhang, "A novel algorithm for inverse Arnold transforms," Journal of Software, vol. 15, no. 10, pp. 1558-1564, 2004 . 
[21] K. Mizuno, K. Takagi, Y. Terachi, S. Izumi, H. Kawaguchi, and M. Yoshimoto, "A sub-100mw dual-core HOG accelerator VLSI for parallel feature extraction processing for HDTV resolution video," IEICE Transactions on Electronics, vol. 96, no. 4, pp. 433443, 2013.

[22] H. Li, Research on quality evaluation method based on the gradient of structural similarity image [Ph.D. thesis], Xi'an University of Science and Technology, Xian, China, 2012.

[23] Vqeg, "Final report from the video quality experts group on the validation of objective models of video quality assessment," 2000, http://www.vqeg.org/. 


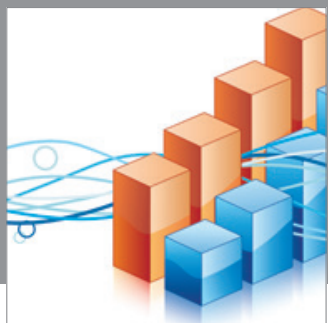

Advances in

Operations Research

mansans

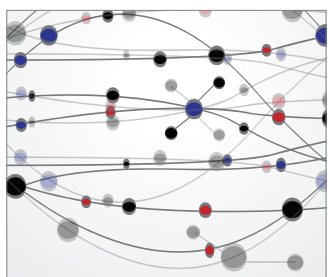

The Scientific World Journal
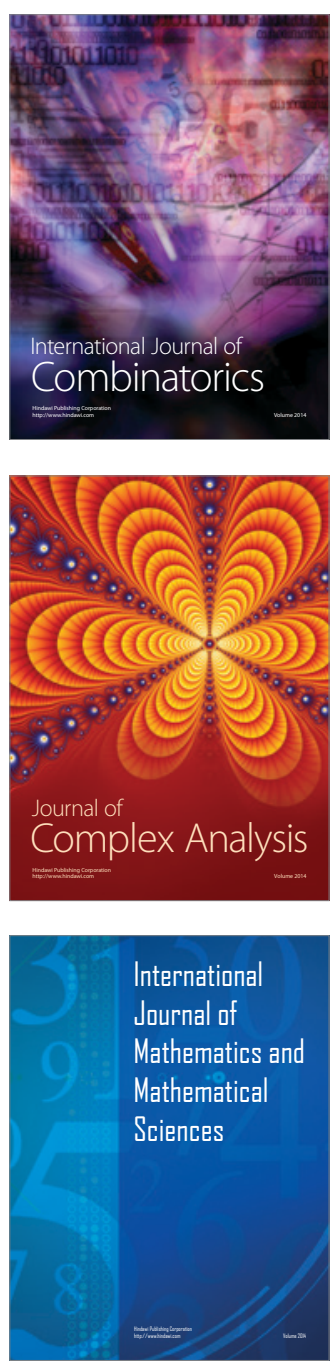
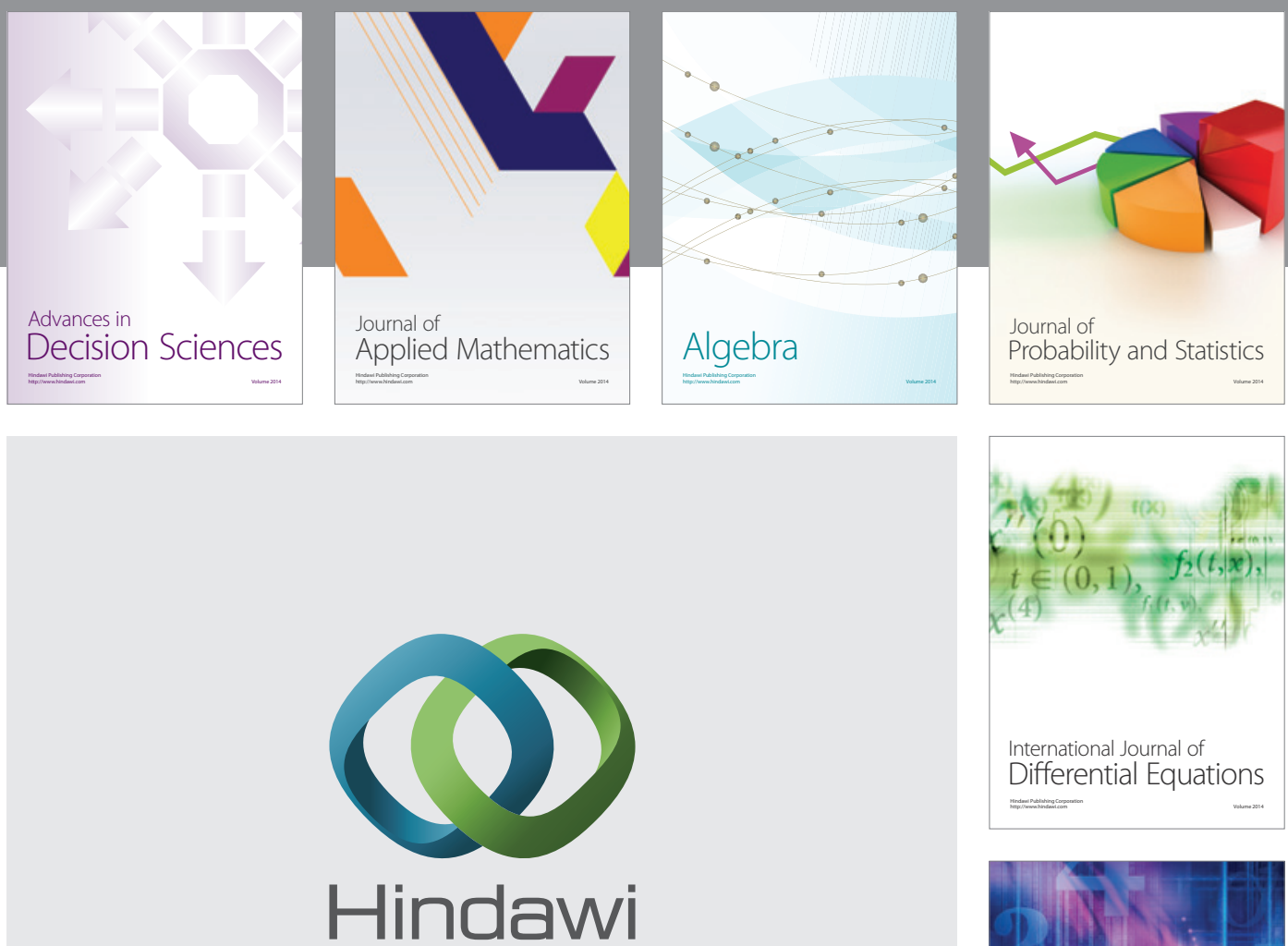

Submit your manuscripts at http://www.hindawi.com
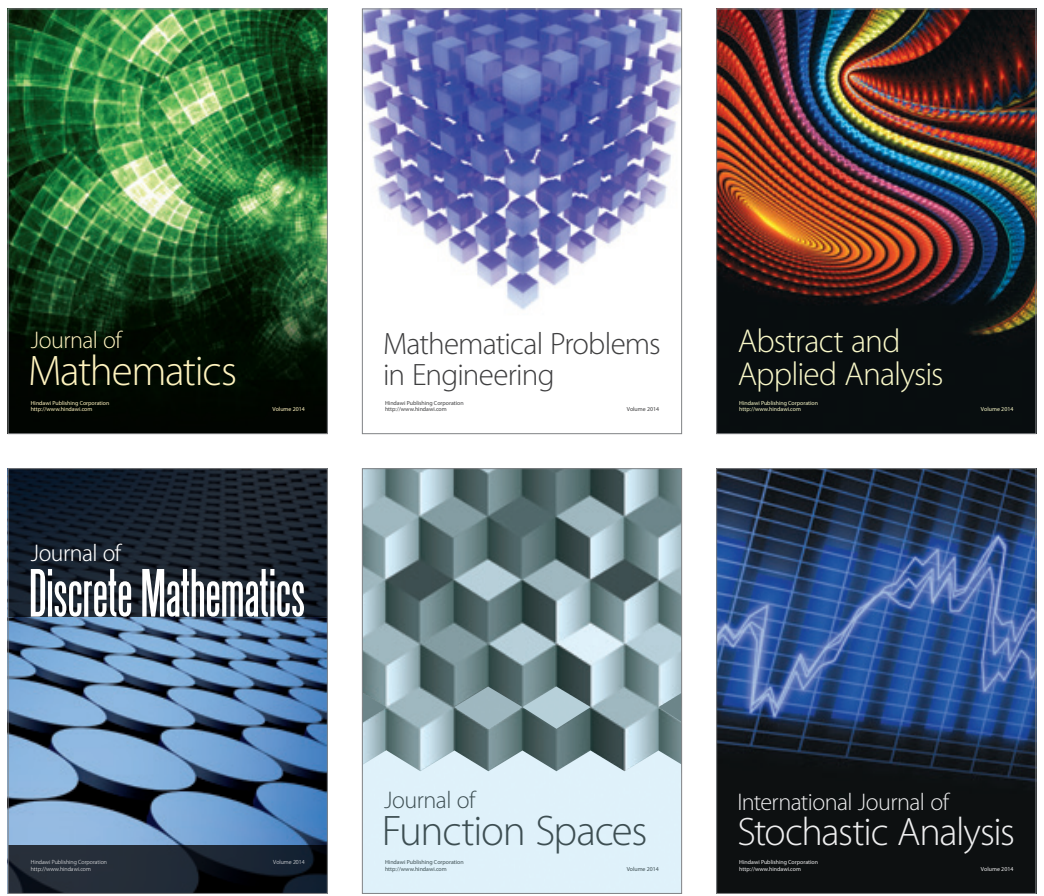

Journal of

Function Spaces

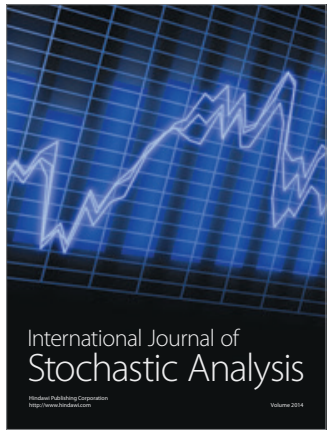

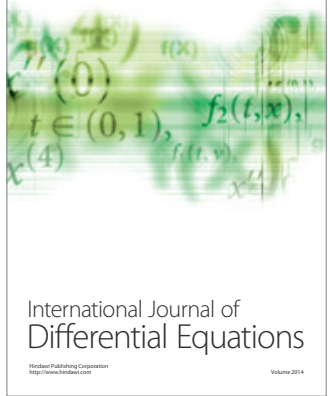
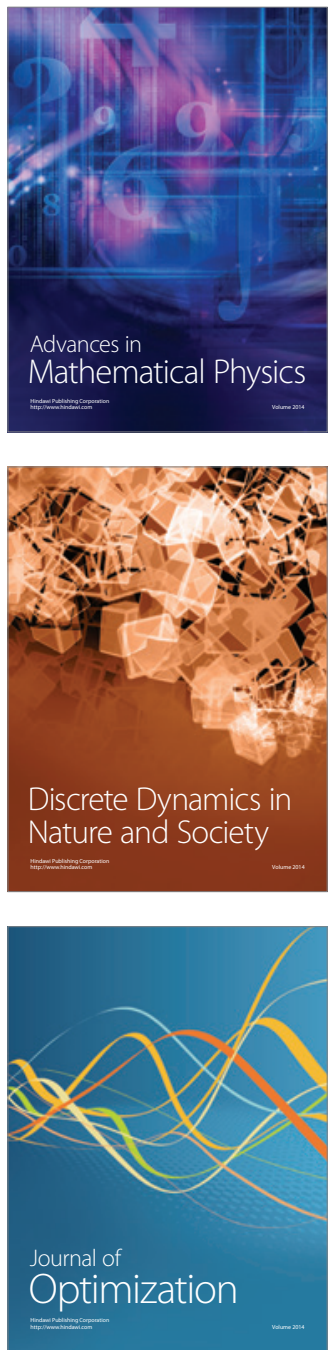